\title{
О некоторых фундаментальных факторах устойчивости системы корпоративного управления
}

\author{
Зенков Олег Сергеевич \\ Certified Internal Auditor \\ Член НКП «Институт внутренних аудиторов» (https://iia-ru.ru/) \\ Москва, Нарышкинская аллея, д. 5, стр. 1 \\ E-mail: ozenkov@mail.ru
}

\begin{abstract}
Аннотация
Многоуровневые системы управления обладают рядом свойств, которые на первый взгляд могут выглядеть парадоксальными. Например, обогащение системы обратными связями не обязательно ведет к повышению устойчивости системы и может даже дестабилизировать ее динамику. Построена математическая модель корпоративного управления, с помощью которой выявляются подходы к обеспечению устойчивости и управляемости компании, анализируются наиболее часто предлагаемые и обсуждаемые новации по организации корпоративного управления, системы внутреннего контроля.

Проведенный анализ модели указывает на опасность чрезмерно сильного «акционерного контроля»: активная ревизионная комиссия, даже работая профессионально, увеличивает нестабильность компании.

Показано также, что одним из важнейших условий устойчивости и управляемости компании является наличие механизмов, обеспечивающих делегирование принятия решений по возможности на все уровни управления: стратегия, предполагающая совершенствование исполнительской дисциплины, рано или поздно приведет к потере управляемости динамической модели компании.

Существенный запас устойчивости многоуровневой системе управления доставляют: участие в построении системы внутреннего контроля всего персонала организации; формирование внутреннего аудита как подразделения, оценивающего систему внутреннего контроля, управления рисками и корпоративного управления (наличие только контрольного подразделения, обеспечивающего обратную связь, фундаментальным фактором устойчивости не является); поддержание максимальной независимости внутреннего аудита и его функциональной подотчетности совету директоров.

Исследованы и интерпретированы факторы, вызывающие попадание компании в ловушку «вблизи границы устойчивости». Как правило, данные факторы характерны для небольших государственных компаний и дочерних компаний холдинговых структур; в рамках рассматриваемой модели данные факторы проявляются в виде отсутствия связей между определенными уровнями управления. Показано, что приоритетность мероприятий по выводу компании из кризиса в такой ситуации играет важнейшую роль: попытка акционера повлиять на ситуацию разумными, зарекомендовавшими себя на практике «точечными» решениями может локально улучшить показатели компании, но оставить ее в ловушке «вблизи границы устойчивости». С позиций обеспечения устойчивости компании анализируются предлагаемые международными стандартами подходы к организации функции внутреннего аудита.
\end{abstract}

Ключевые слова: устойчивость корпоративного управления, многоуровневые системы управления, математическая модель, управляемость компании, внутренний аудит, акционерный контроль

JEL: G34 


\section{Введение}

При построении системы управления рисками и внутреннего контроля в организации принято обращаться к лучшим корпоративным практикам. Данные практики являются квинтессенцией опыта, накопленного при построении функции во множестве различных компаний, и отражены в таких документах, как [Концепция COSO Комитета спонсорских организаций комиссии Тредуэя «Внутренний контроль. Интегрированная модель», 2013; Международные основы профессиональной практики внутреннего аудита, 2017; Кодекс корпоративного управления, 2014; Методические указания по подготовке положения о внутреннем аудите, 2014; Методические рекомендации по организации работы внутреннего аудита в акционерных обществах с участием Российской Федерации, 2014] и др.

Вместе с тем, обоснование используемых принципов исключительно с эмпирических позиций («лучшие практики») содержит определенные подводные камни. Так, время от времени возникают дискуссии о необходимости ослабить (или вовсе исключить) определенные требования стандартов ввиду национальных или отраслевых экономических особенностей. Поскольку исследования, отражающие результаты внедрения обсуждаемых практик, как правило, проводились в других странах без акцентирования на определенной отрасли, аргумент о «лучших корпоративных практиках» не является в данных дискуссиях достаточно убедительным.

Представляется интересным оценить используемые концепции не только с позиций накопленного эмпирического опыта, но также исходя из необходимости построения устойчивой системы корпоративного управления. В случае если определенные условия устойчивости возникают при анализе даже простейших моделей, можно сделать предположение о фундаментальном характере указанных условий и об их высокой значимости при построении системы корпоративного управления на практике - независимо от особенностей экономической среды, в которой находится конкретное предприятие.

Как правило, возможные ограничения в получении информации верхними уровнями управления компании в литературе по корпоративному управлению рассматриваются как безусловно негативный фактор. В качестве примера можно привести ставшее уже классическим обсуждение А. Шейфера и Р.У. Вишни [Shleifer, Vishni, 1997] эффекта концентрации собственности: несмотря на то, что контролирующий акционер в той или иной степени ущемляет права миноритариев, кредиторов и других стейкхолдеров, он обладает значительной мотивацией к сбору и анализу информации и проведению мониторинга менеджмента. Е. Равина и И.П. Сапиенса [Ravina, Sapienza, 2010], анализируя эффективность деятельности независимых директоров, также апеллируют к фактору погруженности члена совета директоров в операционную деятельность компании, негативно оценивая ограничения независимого директора в получении информации.

Большая погруженность верхних слоев управления в дела компании имеет безусловно положительное значение с точки зрения агентской теории: в этой ситуации информированность принципала (акционера) или его представителя (члена совета директоров) ограничивает возможности менеджмента компании в части реализации личных интересов за счет ресурсов компании в случае потери лояльности менеджмента к акционерам.

Вместе с тем, положительность эффекта наращивания интенсивности коммуникаций между отдельными уровнями управления компании / холдинга не представляется настолько безусловной: так, интересным представляется соотнести устойчивость компании как динамической системы с конфигурацией и интенсивностью связей между уровнями управления, характерной для наиболее часто встречающихся моделей корпоративного управления.

Подобный анализ системы корпоративного управления как математического объекта позволит подойти к более или менее определенным ответам на часто встречающиеся следующие вопросы.

- Имеет ли предоставление всем уровням управления дополнительной информации об операционной деятельности безусловно положительный эффект? Какова наиболее приемлемая конфигурация системы обратных связей в системе корпоративного управления?

- Насколько целесообразно активизировать ревизионную комиссию как орган акционерного контроля, увеличив объем (выбрав дополнительные направления) проверочных мероприятий? Какие шаги должен предпринимать акционер по результатам ревизионных проверок? Целесообразно ли акционеру напрямую (минуя управленческую вертикаль) воздействовать на ключевые операционные процессы в компании?

- Что важнее для построения эффективной и устойчивой корпоративной системы - внедрение системы частичного делегирования решений вниз или, напротив, укрепление исполнительской дисциплины на всех уровнях менеджмента?

- Насколько важным является участие операционных менеджеров и линейных сотрудников компании в поддержке системы внутреннего контроля? Возможно, качественной работы контрольно-ревизионного подразделения более чем достаточно?

- Действительно ли обязательна функциональная подотчетность внутреннего аудита совету директоров компании или формирования обратной связи на исполнительный орган вполне достаточно? Или, напротив, нужно ли направлять отчеты внутреннего аудита исполнительному органу ведь можно рассматривать внутренний аудит как «орган совета директоров»? 
- $\quad$ Не является ли декларируемый функционал внутреннего аудита («оценка и повышение эффективности процессов управления рисками, контроля и корпоративного управления») слишком верхнеуровневым? Возможно, эффективнее будет перефокусировать внутренний аудит на проверку финансово-хозяйственной деятельности компании?

Иерархически построенная корпоративная система как сложный объект управления зачастую демонстрирует не очевидную на первый взгляд динамику: отдельные свойства такого объекта могут даже показаться парадоксальными. Эта специфика не всегда очевидна, и предпринимаемые попытки упростить «лучшие корпоративные практики» являются совершенно естественными. Однако мы покажем, что соблюдение «лучших практик» построения системы внутреннего контроля является фундаментальным залогом устойчивости системы корпоративного управления, и попытки ослабить или упростить данные подходы ${ }^{1}$ несут для компании высокий риск потери управляемости и недостижения целевых показателей. Автор выражает искреннюю благодарность Денису Малыхину (Certified Internal Auditor, CIA), д-ру Александру Григорьеву (Maastricht University), Евгению Братцеву, Кириллу Федотову за просмотр рукописи и многочисленные полезные идеи и замечания, которые были учтены в ходе ее доработки.

\section{Базовая модель В.И. Арнольда}

В своих выступлениях и публикациях академик РАН В.И. Арнольд [Арнольд, 2004], упоминая о неустойчивости многоступенчатого управления, неоднократно обращался к следующей простой модели.

Имеется организация, выпускающая продукт $x_{1}$,

руководитель принимает решение, влияющее на скорость производства: $\dot{x}_{1}=x_{2}$. В свою очередь, поведение этого руководителя управляется руководителем более высокого ранга: $\dot{x}_{2}=x_{3}$, и т.д.

Самый высокий руководитель (ранга $n$ ) реализует обратную связь: если поведение всех «промежуточных» руководителей определяется поведением вышестоящих, то заинтересованность руководителя ранга $n$ связана с производственными показателями: он мотивирован достичь выпуска продукта точно в размере $C_{0}: \dot{x}_{n}=-m\left(x_{1}-C_{0}\right), m>0$.

Возникающая система уравнений переписывается в виде линейного дифференциального уравнения относительно выпуска $x_{1}^{(n)}=-m\left(x_{1}-C_{0}\right)$.
Устойчивость стационарного состояния системы $\left(x_{1}=C_{0}, x_{2}=x_{3}=\ldots=x_{n}=0\right)$ определяется тем, отрицательны ли действительные части всех корней характеристического уравнения $t^{n}=-m$.

Корни образуют на плоскости комплексного переменного $t$ вершины правильного $n$-угольника. При $n \geq 3$ хотя бы один корень попадает в область $R e t>0$, что приводит к неустойчивости модели.

Таким образом, «многоступенчатое управление, описываемое нашей моделью при $n \geq 3$, неустойчиво. Двухступенчатое управление приводит к периодическим колебаниям, но не вызывает катастрофического нарастания колебаний, происходящего при трех- $и$ более ступенчатом управлении.

Настоящую устойчивость обеспечивает только одноступенчатое управление, при котором управляющее лицо более заинтересовано в интересах дела, чем в поощрении со стороны начальства» [Арнольд, 2004].

\section{Интерпретация базовой модели в терминах корпоративного управления. Расширение базовой модели}

Интерпретируя данную («базовую») модель в терминах построения корпоративного управления в акционерном обществе (компании), мы можем выделить следующие четыре ключевых уровня принятия решений [5]:

- уровень 4 - акционер;

- $\quad$ уровень 3 - совет директоров

(наблюдательный совет);

- у уровень 2 - исполнительное руководство (генеральный директор);

- уровень 1 - операционный менеджмент, непосредственно занимающийся выпуском продукта.

Стационарное состояние характеризуется стабильным уровнем выпуска $x_{1}=C_{0}$ и нулевыми значениями «управляющих» переменных $x_{2}, x_{3}, x_{4}$. В случае если выпуск $x_{1}$ отклоняется от равновесного $C_{0}$, «управленческие» переменные начинают влиять (с определенной инерцией) на выпуск, что обеспечивает колебания $x_{1}$ вокруг стационарного состояния $C_{0}$. Устойчивые системы характеризуются постепенным сокращением амплитуды данных колебаний; в неустойчивых системах размер колебаний будет экспоненциально расти.

\footnotetext{
${ }^{1}$ Например, положить в основу системы внутреннего контроля в компании деятельность контрольно-ревизионного подразделения, подотчетного только генеральному директору; или рассматривать подразделение внутреннего аудита как «карманный инструмент» совета директоров компании, исключив возможность обсуждения и согласования результатов проверок с объектом аудита и исполнительным руководством компании.
} 
Вместе с тем, необходимо отметить определенные ограничения базовой модели, оставляющие за рамками значимые практические аспекты, актуальные именно для системы корпоративного управления в компании:

1) набор связей между уровнями управления базовой модели достаточно беден: например, не разрешается оказывать управленческое воздействие вниз «через голову»; у менеджмента и совета директоров отсутствует качественная информация о состоянии производства: их мотивация состоит только в удовлетворении запросов непосредственного руководства;

2) любая связь обеспечивает совершенно точную передачу сигнала: искажения при передаче сигнала отсутствуют;

3) менеджерам не делегируется хотя бы частичная возможность принимать самостоятельные решения; они лишь передают управленческие сигналы вниз по иерархии.

За счет обогащения модели дополнительными связями мы исключим ограничение (1) и минимизируем ограничение (3). Влияние искажения сигнала (ограничение (2)) мы обсудим в конце статьи; однако возможные искажения главным образом сформируют неточность при определении объема выпуска, близкого к целевому. Нашей же целью является исследование феномена устойчивости иерархической системы, поэтому в целом мы оставим влияние искажений на целевой выпуск за рамками данной работы. Определяющей чертой рассматриваемой нами модели является наличие определенной «инерционности» в передаче решений: каждый полученный с верхнего или нижнего уровня сигнал влияет лишь в той или иной степени на производную соответствующей управляющей переменной. Как мы покажем далее, именно эта «инерционность» создает для системы корпоративного управления множество интересных и зачастую парадоксальных свойств.

Формально принимаемые решения органов управления носят дискретный характер: так, собрание акционеров происходит, как правило, несколько (или вообще один) раз в год; заседания совета директоров - в среднем один или два раза в месяц. Вместе с тем, неформальная коммуникация акционера и совета директоров с компанией происходит значительно чаще: предоставляются периодическая отчетность компании, результаты проверок контрольно-надзорных и регуляторных органов, появляются публикации в СМИ; контролирующие акционеры (их представители) встречаются и обсуждают насущные вопросы с членами совета директоров и менеджментом компании. В случае если акционер - государство, инициативы в отношении государственных компаний формируются, обсуждаются, изменяются и формулируются в виде поручений практически непрерывно. Кроме того, функционируют комитеты совета директоров, что дополнительно обогащает управленческий поток «информации сверху».

Таким образом, фактический «шаг дискретности» участия акционера и совета директоров в управлении компанией значительно ниже формального. Принятие решений все же остается дискретным (в конце концов, ночью большинство работников и управленцев спят!), но, исходя из изложенного выше, мы считаем возможным аппроксимировать деятельность компании непрерывной моделью. Возможная незначительная разница между поведением траекторий дискретной и «приближающей» ее непрерывной системы не играет значимой роли в поиске качественных характеристик, обеспечивающих устойчивость исследуемой системы.

В дальнейшем мы традиционно говорим о значениях $x_{1}$ как о «выпуске продукции», но возможны и другие интерпретации переменной. В нашем случае разумно интерпретировать $x_{1}$ как «поток прибыли», из которой акционер извлекает дивиденды. Экономика модели носит «эндогенный» характер: предприятие генерирует прибыль исходя из имеющихся ресурсов; возможность наращивания ресурсов (докапитализации и т.д.) отсутствует. В равновесном состоянии поток прибыли $x_{1}=C_{0}$ стабилен ${ }^{2}$. Исходя из этих условий акционер заинтересован в стабильном потоке дивидендов: так, превышение прибыли над равновесным уровнем $C_{0}$ ему невыгодно, поскольку может привести к преждевременному истощению ресурсов и декапитализации предприятия.

Параметр $m$ характеризует состояние «акционерного контроля»: наличие обратной связи о выпуске акционеру (отчетность, результаты мониторинга, содержательные акты ревизионной комиссии).

Для холдинговой структуры количество звеньев управления растет: как правило, в случае достаточного контроля над дочерней структурой головная организация формирует значительную часть совета директоров дочернего предприятия из менеджмента (сотрудников) головной организации. Управленческое воздействие от головного акционера должно сначала пройти ряд управленческих уровней головной организации, затем попадает в совет директоров дочерней компании и далее начинает «спускаться» по уровням управления дочерним предприятием: цепочка передачи управленческих сигналов удлиняется. Там, где это возможно, мы попробуем получить результаты в самом общем виде (для $n>4$ ), имея в виду возможность их интерпретации для холдинговых структур.

В данной статье мы рассмотрим различные варианты обогащения базовой модели дополнительными (прямыми и обратными) связями и попробуем выявить фундаментальные принципы их формирования и

2 В случае инфляции - стабилен в реальных (скорректированных на инфляцию) величинах. 
мониторинга, обеспечивающие устойчивость системы корпоративного управления.

Система линейных уравнений для базовой модели $\dot{x}=A x+C(x, C-$ векторы размерности $n$;

$A$ - матрица $n \times n)$ характеризуется матрицей $A$, имеющей единицы над главной диагональю и ненулевой элемент $a_{n 1}=-m$ (остальные элементы матрицы $A$ нулевые), а также вектором

$$
C=\left(\begin{array}{c}
0 \\
\ldots \\
0 \\
-m C_{0}
\end{array}\right) .
$$

Для рассмотрения возможностей стабилизации системы мы введем дополнительный вектор управления $u: \dot{x}=A x+C+u$. Поскольку наблюдаемыми являются только переменные $x_{1}, \ldots, x_{n}$ (а не их производные), и система должна сохраниться замкнутой, мы предполагаем $u=B x$.

Новая система по-прежнему линейна: $\dot{x}=\tilde{A} x+C$, где $\tilde{A}=A+B$, при этом матрица $B$ обогащает систему дополнительными связями между уровнями управления.

Пример. Рассмотрим модель акционерного общества $(n=4)$. Добавляя в исходную матрицу $A$ элемент $b_{12}$, мы получим обогащенное уравнение $\dot{x}_{1}=x_{2}+b_{13} x_{3}$, теперь динамика операционного уровня $x_{1}$ в каждый момент определяется не только состоянием исполнительного руководства $x_{2}$, но и состоянием совета директоров $x_{3}$ : появилось отсутствовавшее в базовой модели влияние совета директоров на операционный менеджмент.

Дополнив матрицу, например, элементом $b_{31}$ мы получим обогащенное уравнение $\dot{x}_{3}=b_{31} x_{1}+x_{4}$ : на динамику состояния совета директоров теперь влияет не только акционер (как в базовой модели), но и состояние выпуска $x_{1}$. В модели появляется обратная связь «с операционного уровня».

Добавляя в матрицу $\tilde{A}$ ненулевые диагональные элементы, мы допускаем возможность «самокорректировки» управленческих переменных. Например, в случае $b_{22} \neq 0 \quad \dot{x}_{2}=b_{22} x_{2}+x_{3}$ динамика исполнительного руководства (уровень 2) определяется не только «указаниями» совета директоров $x_{3}$, но и реагированием на свое собственное состояние. Возможность такой «самокорректировки» можно интерпретировать как делегирование данному уровню управления определенной самостоятельности, и далее мы увидим, что предоставление такой возможности играет значительную роль в построении устойчивой системы управления.
Затем мы будем исследовать конфигурации матрицы $\tilde{A}$, характеризующиеся устойчивостью стационарных решений системы $\dot{x}=\tilde{A} x+C$.

Элементы $\tilde{A}$ мы обозначим как $\left(a_{i j}\right)$; разумно предположить, что при $i>j$ (элементы выше диагонали) $a_{i j} \geq 0$ начальники оказывают управленческое воздействие «вниз», возможно, минуя несколько уровней управления. Элементы $a_{i j}$ на диагонали и ниже, напротив, не положительны: они характеризуют обратную связь от нижних к более высоким уровням управления (элементы на диагонали - «самокорректировку» соответствующей переменной).

Траектория решения $x(t)$ системы $\dot{x}=\tilde{A} x+C$ может за счет внешних возмущающих воздействий отклоняться от стационарного решения $x_{0}(t)=\left(C_{0}, 0, \ldots, 0\right)$. Асимптотическая устойчивость решения предполагает, что в случае если в момент времени $t_{0}$ произошло внешнее воздействие (отклонение) $\left(\varepsilon_{1}\left(t_{0}\right), \ldots, \varepsilon_{n}\left(t_{0}\right)\right)$, то «возмущенное» решение $x_{\varepsilon}(t)=\left(C_{0}+\varepsilon_{1}(t), \ldots, \varepsilon_{n}(t)\right)$ стремится к стационарному состоянию:

$\lim _{t \rightarrow+\infty}\left\|x_{\varepsilon}(t)-x_{0}(t)\right\|=0$, где $\|\cdot\|-$

норма, принятая в пространстве решений системы (например, евклидова норма).

Условия устойчивости системы $\dot{x}=\tilde{A} x+C$ совпадают с условиями устойчивости для однородной системы $\dot{x}=\tilde{A} x$. Условия асимптотической устойчивости, применяемые далее в данной статье, включают:

- $\quad$ необходимое условие - строгая положительность всех коэффициентов $a_{k}$ характеристического многочлена системы;

- $\quad$ необходимое и достаточное условие - отрицательность вещественных частей всех собственных чисел матрицы $\tilde{A}$;

- $\quad$ необходимое и достаточное условие (критерий Гурвица $\left.{ }^{3}\right)$ при $n=4$ : для коэффициентов характеристического многочлена $a_{4} t^{4}+\ldots+a_{1} t+a_{0}$ должно выполняться соотношение $a_{1}\left(a_{3} a_{2}-a_{4} a_{1}\right)-a_{0} a_{3}^{2}>0$ (при всех строго положительных $\left.a_{k}\right)$.

Пример. Рассмотрим ситуацию, когда на динамику выпуска влияют все переменные системы (включая сам выпуск): $\dot{x}_{1}=-\alpha\left(x_{1}-C_{0}\right)+x_{2}+\beta x_{3}+\gamma x_{4}$, а остальные уравнения системы не отличаются от базовой модели. Характеристический многочлен системы $t^{4}+\alpha t^{3}+m \gamma t^{2}+m \beta t+m$, и условие Гурвица переписываются в виде $m \beta(\alpha m \gamma-m \beta)-m \alpha^{2}>0$, или $\gamma>\frac{\alpha^{2}+\beta^{2} m}{\alpha \beta m}$ :

\footnotetext{
${ }^{3}$ Формулировку критерия Гурвица для произвольного $n$, а также другие подходы к определению устойчивости можно найти, например, в [Бесекерский, Попов, 2003].
} 
для устойчивости необходимо сильное влияние акционера на операционные процессы, характеризующееся коэффициентом $\gamma$.

Область устойчивости произвольной матрицы $\tilde{A}$ вряд ли можно описать в интерпретируемых терминах для произвольного $n$. Далее зачастую мы ограничиваемся рассмотрением случаев $n=4$ (отдельное акционерное общество), и даже для этого случая появляется множество вариантов конфигураций элементов матрицы. Вариант системы, рассмотренный в примере выше, теоретически имеет право на существование наравне с другими конфигурациями, но на практике вряд ли может встретиться. В дальнейшем мы сосредоточимся на условиях устойчивости для нескольких наиболее часто обсуждаемых конфигураций и проинтерпретируем их в терминах корпоративного управления.

\section{Параметры модели как характеристики системы внутреннего контроля компании}

Элементы матрицы $\tilde{A}$ обеспечивают связи между состояниями уровней управления и выпуском продукта в системе $\dot{x}=\tilde{A} x$ и полностью определяют динамику системы после появления «внешних» возмущений (отклонений выпуска / одной или нескольких управленческих переменных).

Покажем, что рассматриваемая модель корпоративного управления имеет все основные черты, характеризующие систему внутреннего контроля. Компоненты внутреннего контроля, определяемые Концепцией $\mathrm{COSO}$ «Внутренний контроль. Интегрированная модель» (2013), интерпретируются для модели следующим образом.

Контрольная среда. Модель доставляет набор стандартов, процессов и структур, обеспечивающих согласованные действия всех уровней управления для сохранения выпуска $x_{1}$ на целевом уровне $C_{0}$. Сохранить выпуск в районе целевого сможет только устойчивая система.

Оиенка рисков. Характерным для рассматриваемой модели риском является непредсказуемое внешнее событие, которое влечет за собой отклонение выпуска $x_{1}$ от целевого уровня, или отклонение (ошибку) на некоторых уровнях управления («ошибка менеджера»).

Контрольные процедуры. Связи между уровнями управления, определяемые элементами матрицы $\tilde{A}$, обеспечивают реагирование на произошедшее отклонение (риск). Кроме того, факторы реагирования на риск могут присутствовать и на том же уровне управления, где произошло отклонение: так, далее мы говорим о важности существования контрольных механизмов операционного уровня.

Информация и коммуникации. Возможность коммуникации между уровнями управления и внутри каждого уровня обеспечивается структурой и сбалансированностью набора связей, определяемых матрицей $\tilde{A}$.

Процедуры мониторинга. Только обогащения модели связями далеко не достаточно для обеспечения устойчивости модели: крайне важна также сбалансированность этих связей. Можно предположить, что на практике внешние возмущения могут влиять не только на состояние переменных модели, но и на интенсивность связей модели. Таким образом, наличие «внешнего» по отношению к операционной модели мониторинга параметров модели крайне важно для проведения при необходимости корректировок параметров системы внутреннего контроля, обеспечивающих устойчивый выпуск, сохраняющийся около целевого уровня.

\section{Интерпретация устойчивости в терминах корпоративного управления}

\section{Делегирование решений и контрольные механизмы операционного уровня}

Утверждение 1. В случае если в матрице $\tilde{A}$ все диагональные элементы $a_{i i}=0$ (самокорректировка переменных $x_{1}, \ldots, x_{n}$ отсутствует), асимптотическая устойчивость системы не достигается.

$\gg$ У характеристического многочлена матрицы $\tilde{A}$ коэффициент при $t^{n-1}$ равен $(-1)^{n-1} \operatorname{tr} \tilde{A}$, где след матрицы $\operatorname{tr} \tilde{A}=0$ (все диагональные элементы $\tilde{A}$ нулевые). Таким образом, в уравнении выпуска $x_{1}^{(n)}+a_{n-1} x^{(n-1)}+\ldots+a_{1} x^{(1)}+m\left(x_{1}-C_{0}\right)=0$

коэффициент $a_{n-1}=0$ : необходимое условие асимптотической устойчивости не выполняется. 口

Утверждение 1 показывает, что для устойчивости модели необходимо наличие «самокорректировок» хотя бы одной переменной модели. Можно интерпретировать самокорректировку менеджера определенного уровня как возможность принятия им хотя бы частично самостоятельных решений. Тогда утверждение 1 можно интерпретировать следующим образом.

В отсутствие разумной системы делегирования принятия решений «вниз» совершенствование исполнительской дисииплины в компании ведет к потере управляемости 4 .

Наиболее важной на практике представляется возможность самокорректировки выпуска $x_{1}$ : коэффициент $a_{11}>0$ характеризует влияние отклонения выпуска на скорость изменения самого выпуска. Коэффициент $a_{11}$, отражающий степень инертности операционных процессов по отношению к отклонениям, естественно интерпретировать как наличие

${ }^{4}$ Данный вывод действует не только в корпоративной среде и актуален для любой иерархической системы (например, для армии). 
механизмов управления рисками (контрольных процедур) на уровне операционных процессов компании. Таким образом, можно сделать следующий вывод: необходимым условием устойчивости системы корпоративного управления является наличие контрольньх механизмов, обеспечивающих «самоконтроль» хотя бы на одном из уровней управления. Наиболее важным на практике представляется наличие контрольных механизмов на уровне операционных процессов, направленных на сокращение рисков отклонения выпуска от иелевого.

Данное требование само по себе не обеспечивает устойчивость системы корпоративного управления, но в случае его невыполнения модель становится неуправляемой. В дальнейшем, если не оговорено обратное, будем всегда предполагать, что $a_{11}>0$.

Требование существования контрольных механизмов операционного уровня как одного из элементов системы внутреннего контроля в компании подчеркивает важное значение участия сотрудников структурных подразделений в обеспечении функционирования системы внутреннего контроля в компании. Случается, что на практике данному требованию не уделяется должного внимания: вместе с тем, как мы видим, для устойчивости системы корпоративного управления оно является одним из определяющих. Отметим, что широко используемое определение внутреннего контроля, предоставляемое концепцией COSO «Внутренний контроль. Интегрированная модель» (2013), также подчеркивает роль сотрудников операционного уровня в организации системы внутреннего контроля.

Внутренний контроль - это проиесс, осуществляемый советом директоров, менеджментом и другим персоналом организации, направленный на обеспечение разумной уверенности в достижении иелей, связанных с операционной деятельностью, подготовкой отчетности и комплайенс.

\section{Сбалансированность системы внутрен- него контроля в акционерном обществе}

Необходимо отметить, что требование строгой положительности коэффициента характеристического многочлена при $t^{k}$ хотя в общем случае и может быть обеспечено сложной комбинацией дополнительных каналов управленческого воздействия и обратных связей, надежнее всего обеспечивается требованием отличия от нуля параметра $a_{k 1}$, характеризующего обратную связь с операционного уровня («выпуск» $\left.x_{1}\right)$ на уровень управления $k$.

Какой бы уровень управления мы ни рассмотрели, информация об операционных показателях занимает особое положение среди обратной связи, получаемой менеджментом этого уровня:

- значения выпуска $x_{1}$, в отличие от «управленческих» переменных $x_{k}$, на практике являются хорошо наблюдаемыми и объективно измеримыми;
- $\quad$ значения выпуска характеризуют достижимость важнейших операционных и стратегических целей компании.

Как правило, на практике обсуждение возможностей получения менеджментом оперативной и неискаженной обратной связи акцентируется на получении управленцем данных о производственных (операционных) показателях компании. Обычно дискуссия касается возможности формирования такой связи: неявно предполагается, что для любого уровня управления «чем больше обратной связи, тем лучше» (одним из примеров являются дискуссии и мероприятия последних лет, направленные на активизацию деятельности ревизионных комиссий как органа акционерного контроля в государственных компаниях). Рассмотрим, при каких соотношениях параметров обратной связи с операционного уровня модель корпоративного управления демонстрирует устойчивость.

Утверждение 2. В случае если обратная связь в акционерном обществе формируется на всех уровнях управления исходя из отклонения выпуска от стационарного значения

$\dot{x}_{1}=x_{2}-a\left(x_{1}-C_{0}\right)$,

$\dot{x}_{2}=x_{3}-b\left(x_{1}-C_{0}\right)$,

$\dot{x}_{3}=x_{4}-c\left(x_{1}-C_{0}\right)$,

$\dot{x}_{4}=-m\left(x_{1}-C_{0}\right)$,

область асимптотической устойчивости определяется соотношением $0<m<\frac{c}{a}\left(b-\frac{c}{a}\right)$.

$\triangleright$ Рассмотрим характеристический многочлен системы $t^{4}+a t^{3}+b t^{2}+c t+m$, тогда критерий Гурвица выглядит как $c(a b-c)-a^{2} m>0$ (все коэффициенты $a, b, c, m>0)$.

Коэффициенты данной системы могут быть легко интерпретированы:

- $\quad$ как упоминалось выше, коэффициент $a$ характеризует качество контрольных механизмов на уровне операционных процессов организации (практика обсуждения результатов контрольных мероприятий с объектом аудита также положительно отражается на его величине);

- $\quad b, c$ - «количество информации» от контрольного подразделения и в управленческой отчетности, которая по результатам проверочных мероприятий попадает к исполнительному руководству и совету директоров соответственно;

- $m$ - величина обратной связи «на акционера» (акционерный контроль: акты ревизионной комиссии, отчетность перед акционером).

Попробуем интерпретировать условия устойчивости $0<m<\frac{c}{a}\left(b-\frac{c}{a}\right)$. 
1) Необходимо, чтобы все коэффициенты $a, b, c$ были положительны: имеются качественные контрольные механизмы на уровне операционных процессов; отчеты контрольного подразделения после обсуждения с объектом аудита попадают как к исполнительному менеджменту, так и к совету директоров. Данное требование вполне согласуется с существующими лучшими практиками.

2) Параметры $a, c$, характеризующие качество операционных контрольных механизмов и интенсивность обратной связи на совет директоров, влияют на устойчивость системы только в виде соотношения $c / a$ : таким образом, в случае проведения преобразований в компании фокусирование на одновременном улучшении именно этих двух параметров вряд ли разумно.

3) Риск появления неустойчивости растет в случаях, если совет директоров слишком пассивный ( $c / a$ мало) или, напротив, слишком активный ( $c / a$ сравнимо с $b$ или превышает его). У системы, характеризуемой сокращением параметров обратной связи по мере роста уровней управления, больше шансов оказаться устойчивой (и этот факт имеет смысл принимать во внимание при практическом построении системы корпоративного управления), но в целом конфигурация параметров обратной связи в устойчивой системе может быть совершенно разной. Сокращение интенсивности информационных потоков может происходить в том числе и естественным образом, за счет повышения материальности ${ }^{5}$ «подаваемой наверх» информации.

4) В общем случае высокая степень акционерного контроля $m$ создает предпосылки для дестабилизации компании: оптимальной является незначительная (но ненулевая) степень акционерного контроля. Чрезмерно активная ревизионная комиссия может дестабилизировать компанию.

5) Активный акционерный контроль в особенности противопоказан «благополучным» компаниям со зрелой системой внутреннего контроля на операционном уровне ( $а$ сравнительно велико): операционный менеджмент своевременно и качественно устраняет отклонения, не дожидаясь реакции со стороны вышестоящего руководства.

\section{Ревизионная комиссия и ее влияние на устойчивость управления}

Периодически возникает дискуссия о целесообразности расширения функционала ревизионной комиссии: будучи подотчетной акционеру и осуществляя обратную связь на самый высокий уровень (акционерный контроль), ревизионная комиссия может без конфликта интересов оценивать не только вопросы финансово-хозяйственной деятельности, но также деятельность исполнительных органов и совета директоров компании.

В случае если ревизионная комиссия отчитывается акционеру не только об отклонении выпуска $x_{1}$, но и об отклонениях других управляющих переменных $x_{2}, x_{3}$, динамика «переменной акционера» $x_{4}$ определяется комбинацией $-m\left(x_{1}-C_{0}\right)-q x_{2}-r x_{3}$.

Утверждение 3. Расширение функционала ревизионной комиссии в виде $\dot{x}_{4}=-m\left(x_{1}-C_{0}\right)-q x_{2}-r x_{3}$ само по себе устойчивость системы не обеспечивает.

$\triangleright$ Система уравнений перепишется в виде:

$\dot{x}_{1}=x_{2}-a\left(x_{1}-C_{0}\right)$,

$\dot{x}_{2}=x_{3}$,

$\dot{x}_{3}=x_{4}$,

$\dot{x}_{4}=-m\left(x_{1}-C_{0}\right)-q x_{2}-r x_{3}$.

Рассмотрим характеристический многочлен $t^{4}+a t^{3}+r t^{2}+(q+r a) t+(m+q a)$

и соответствующий критерий устойчивости Гурвица: $(q+r a)[a r-(q+r a)]-a^{2} m>0$, или $-q(q+r a)-a^{2} m>0$.

Коэффициенты $q, r, a, m>0$, поэтому система не имеет решений. $\square$

Таким образом, решение об обогащении функционала ревизионной комиссии не приближает нас к появлению устойчивости системы - ни при каком распределении усилий ревизионной комиссии между мониторингом и оценкой финансово-хозяйственной деятельности / исполнительного руководства / совета директоров! Усиление ресурсов ревизионной комиссии также не приведет к желаемому результату.

Мы можем видеть, что нередко встречающееся ироничное отношение к деятельности ревизионных комиссий имеет под собой определенные основания. Слабость механизма акционерного контроля обусловливается в первую очередь даже не ограниченностью ресурсов ревизионной комиссии, а специфической ролью акционерного контроля в структуре корпоративного управления. Даже при наличии эффективных контрольных механизмов операционного уровня сам по себе ревизионный контроль обеспечить устойчивость компании не способен.

\footnotetext{
${ }^{5}$ Так, Р. Экклес и Т. Юманс [Eccles, Youmans, 2015] характеризуют материальность информации, направляемой советом директоров стейкхолдерам компании следующим образом: «...материальность специфична по отношении к компании, аудитории и ситуации. Важнейший принцип материальности - осознанность. Советы директоров, которые достаточно храбры для того, чтобы быть точными и ограничить отчетность только материальной информацией, направляют сигнал о том, что они действительно способны проделать это упражнение - другими словами, могут управлять».
} 


\section{Попадание компании в «ловушку колебательного режима»}

Рассмотрим также вопрос о возможности длительного нахождения системы в колебательном режиме с постоянной амплитудой. В этом случае один или несколько корней характеристического уравнения системы $t^{4}+a_{3} t^{3}+a_{2} t^{2}+a_{1} t+a_{0}=0$ имеют чисто мнимый вид $t=s i, s \in R$ (а действительные части остальных корней отрицательны). Чисто мнимый вид корни данного характеристического уравнения могут принять только все сразу (сопряженные корни вида $\pm \alpha i, \pm \beta i)$, при этом должны выполняться следующие условия: $a_{1}, a_{3}=0, a_{0}, a_{2}>0, a_{2}^{2}-4 a_{0} \geq 0$.

Как уже отмечалось в утверждении 1 , равенство нулю коэффициента $a_{3}=\operatorname{tr} \tilde{A}=a_{11}+a_{22}+a_{33}+a_{44}$ равносильно требованию равенства нулю диагональных элементов матрицы $\tilde{A}$.

В этом случае коэффициент

$a_{2}=\sum_{1 \leq i<j \leq 4}\left(a_{i i} a_{j j}-a_{i j} a_{j i}\right)=-\sum_{1 \leq i<j \leq 4} a_{i j} a_{j i} \geq 0$,

поскольку элементы по разные стороны от диагонали $\tilde{A}$ имеют разные знаки (или обращаются в ноль). Для положительности $a_{2}$ достаточно существования двух симметричных относительно диагонали ненулевых элементов; для положительности $a_{0}=\operatorname{det} \tilde{A}-$ вообще говоря, четырех ненулевых элементов матрицы $\tilde{A}$. Существует всего три конфигурации «колебательных» систем, имеющих четыре ненулевых элемента. Каждая из них носит вырожденный характер: операционный уровень $x_{1}$ взаимодействует с одним из управленческих уровней $x_{i}$ по «модели колебаний» $\dot{x}_{1}=a_{1 i} x_{i}, \dot{x}_{i}=-a_{i 1}\left(x_{1}-C_{0}\right)$; оставшиеся два уровня взаимодействуют аналогично и независимо от предыдущей пары уровней.

Более интересным представляется следующий вопрос: какое минимальное количество связей в системе можно обнулить с тем, чтобы выйти на режим колебаний при достаточно общих условиях на остальные связи? Проведенный на компьютере перебор вариантов показал, что в режим колебаний можно попасть при обнулении минимум восьми элементов матрицы $\tilde{A}$ (из них четыре элемента - диагональные). Существует ровно девять конфигураций матрицы $\tilde{A}$ с восемью нулевыми коэффициентами, доставляющих системе режим колебаний. Оставаясь в рамках осмысленных интерпретаций, мы продолжаем предполагать, что $a_{12}, a_{23}, a_{34}>0$ (каждый начальник имеет возможность непосредственно влиять на непосредственного подчиненного): этому требованию удовлетворяют только две конфигурации из выявленных. Приведем их здесь (как и в базовой модели, принимаем $\left.a_{12}, a_{23}, a_{34}=1\right)$.
Утверждение 4. Минимальное количество элементов невырожденной матрицы $\tilde{A}$, которое необходимо обнулить для обеспечения $a_{1}, a_{3}=0$, равно 8 . Если $a_{12}, a_{23}, a_{34}=1$, матрица $\tilde{A}$ будет иметь одну из следующих двух конфигураций:

$\begin{array}{cc}\text { Конфигурачия } 1 & \text { Конфигуращия } 2 \\ \left(\begin{array}{cccc}0 & 1 & 0 & * \\ * & 0 & 1 & 0 \\ 0 & * & 0 & 1 \\ * & 0 & * & 0\end{array}\right) & \left(\begin{array}{llll}0 & 1 & * & * \\ * & 0 & 1 & * \\ 0 & 0 & 0 & 1 \\ 0 & 0 & * & 0\end{array}\right) .\end{array}$

Замечание 1. Случай конфигурации 2 является вырожденным и не представляет практического интереса: акционер и совет директоров взаимодействуют друг с другом по классической модели колебаний $\dot{x}_{3}=a_{34} x_{4}, \dot{x}_{4}=-a_{43} x_{3}$, и их динамика не зависит от состояния исполнительного руководства / выпуска. Далее мы ограничимся рассмотрением конфигурации 1.

Замечание 2. Коэффициенты $a_{2}, a_{0}$ характеристического уравнения для конфигурации 1 :

$a_{2}=a_{14} a_{41}+a_{21}+a_{32}+a_{43}>0$
$a_{0}=\left(a_{41}+a_{21} a_{43}\right)\left(1+a_{14} a_{32}\right)>0$

Предположим, что нулевые связи-элементы матрицы $\tilde{A}$ всегда остаются нулевыми. Тогда область в пространстве ненулевых элементов матрицы $\tilde{A}$, определяемая оставшимся условием $a_{2}^{2}-4 a_{0} \geq 0$, имеет, вообще говоря, ненулевую меру. В случае если параметры системы определяются точкой, лежащей «глубоко» в данной области, можно ожидать длительного сохранения колебательного режима - даже несмотря на возможное, осознанное или случайное изменение величины ненулевых связей между уровнями управления.

Рассмотрим конфигурацию корпоративного управления, изложенную в утверждении 4 (конфигурация 1). Основные черты соответствующего акционерного общества:

1) работа совета директоров носит пассивныци, «технический» характер: совет директоров лишь транслирует генеральному директору решения и поручения акционера, получая от него же сигналь общего характера о состоянии дел и не погружаясь в вопросы финансово-хозяйственной деятельности предприятия

2) контрольные механизмы на операционном уровне практически не работают. Генеральный директор формирует для себя обратную связь с операционного уровня - возможно, через деятельность «карманного» контрольно-ревизионного подразделения, не взаимодействующего с операционным менеджментом и советом директоров;

\footnotetext{
6 Это могут быть «отфильтрованные» генеральным директором верхнеуровневые отчеты о состоянии предприятия. Как правило, рассмотрение соответствующего вопроса отражается в протоколе совета директоров формулировкой «Принять к сведению» с последующим единогласным голосованием. Дополнительным негативным сигналом служит факт проведения соответствующего заседания совета директоров в заочном формате.
} 
3) акиионер получает информацию об операционных показателях компании (ревизионная комиссия) $u$ формирует указания операционному менеджменту напрямую, минуя совет директоров и исполнительное руководство.

На практике такая ситуация нередко возникает, например, в государственных компаниях небольшого и среднего масштаба, а также в дочерних компаниях крупных холдинговых структур:

- большинство в совете директоров компании составляют государственные чиновники / менеджмент головной организации, что обеспечивает «технический» характер совета директоров (зачастую государственный чиновник / менеджер головной организации входит сразу во множество советов директоров, работа в советах директоров ему не оплачивается и, как правило, является «дополнительной нагрузкой» к его основной деятельности);

- акционер, понимая неудовлетворительное состояние дел в компании, периодически проводит ротацию «первых лиц». Каждый новый руководитель, стремясь оперативно войти в курс дел, в первую очередь начинает настраивать обратную связь «на себя» (формирование системы внутреннего контроля даже на операционном уровне процесс достаточно длительный). Операционный менеджмент и сотрудники в такой ситуации не спешат брать на себя ответственность за принимаемые решения, сосредоточившись на аккуратном и оперативном исполнении решений руководителя. Такая практика управления постепенно укрепляется и может стать привычным атрибутом управления компанией;

- акционер, получая информацию о неудовлетворительных операционных показателях деятельности, обладая хорошей экспертизой и стратегическим видением ситуации, стремится напрямую повлиять хотя бы на ключевые операционные процессы: формируются директивы акционера, оперативно спускаемые на нижние уровни управления и обязательные к исполнению.

В этой ситуации все участники действуют достаточно разумно (хотя и несколько предсказуемо), но именно попытка акционера повлиять на ситуацию «точечными» решениями (смена руководства, формирование директив), игнорируя необходимость построения устойчивого корпоративного управления, создает для компании «ловушку». При этом компания в той или иной мере устойчива (особенно при определенной поддержке со стороны акционера), хотя и не способна обеспечить стабильного достижения целевых показателей. Поскольку время от времени целевые показатели компанией достигаются (и даже перевыполняются), решения об инвестициях в трансформацию системы управления могут не приниматься достаточно долго.

Вместе с тем, накапливающиеся ошибки управления и нахождение на границе устойчивости доставляют компании слабую степень управляемости. Можно видеть, что первоочередной мерой является замена «технического» совета директоров на работающий ${ }^{7}$, при этом необходимо:

- обеспечить его качественной обратной связью в части операционных процессов (в том числе за счет взаимодействия совета директоров с контрольным подразделением, если таковое имеется);

- совету директоров первоочередное внимание уделить организации системы внутреннего контроля на операционном уровне.

Вместо этого зачастую акционер инициирует проведение тщательной ревизионной проверки организации и, по результатам проверки, приступает к самостоятельной выработке решений, направленных на стабилизацию выпуска. В структуре модели такие решения отражаются в росте соответственно элементов $a_{14}, a_{41}$. Поскольку $a_{2} \sim a_{14} a_{41}, a_{0} \sim a_{14} a_{41}$, эти решения, как правило, увеличивают величину $a_{2}{ }^{2}-4 a_{0}$ : система еще глубже сдвигается в область, соответствующую режиму колебаний.

В подобной ситуации нахождение компании в режиме колебаний - не самый худший сценарий, но достижение по-настоящему важных результатов (управляемость компании, стабилизация выпуска на целевом уровне) оно не обеспечивает.

Время от времени также возникают дискуссии, должно ли контрольное подразделение направлять отчеты совету директоров (или достаточно отчетности руководству компании). Можно видеть, что использование контрольного подразделения только как «инструмента генерального директора» повышает риск возникновения дисбаланса обратных связей и попадания компании в «ловушку» на границе устойчивости.

\section{Внутренний аудит как фундаменталь- ный фактор устойчивости организации}

Международные основы профессиональной практики внутреннего аудита (МОПП) (2017) дают внутреннему аудиту следующее определение.

Внутренний аудит является деятельностью по предоставлению независимых и объективных гарантий и консультаций, направленной на совершенствование работы организации. Внутренний аудит помогает организации достичь поставленных целей, используя

\footnotetext{
${ }^{7}$ Принятию соответствующих решений, как правило, препятствует не только сложность подбора в совет директоров команды профессионалов, но и необходимость понести дополнительные расходы на обеспечение деятельности и на компенсацию членам совета директоров.
} 
систематизированный и последовательный подход к оценке и повышению эффективности процессов управления рисками, контроля и корпоративного управления.

Практическая применимость определения МОПП иногда подвергается сомнению. Функционал внутреннего аудита, предлагаемый определением, характеризуется оппонентами как искусственный; деятельность по оценке системы управления рисками и внутреннего контроля, корпоративного управления - как чрезмерно «верхнеуровневая». Как правило, в качестве альтернативы предлагается рассмотреть формирование объективной и качественной «обратной связи» как основную задачу внутреннего аудита: внутренний аудит должен выявлять конкретные недостатки, неэффективности и риски операционной модели, а также существенные нарушения в деятельности выбранного объекта аудита и отчитываться «наверх» о результатах проверочных мероприятий.

Вместе с тем, рассмотренные нами модели показывают, что наличия качественной обратной связи для устойчивости управления может быть совсем недостаточно: более того, качественная, но несбалансированная по уровням управления обратная связь может привести к дестабилизации компании. Необходимо обеспечить периодическую оценку «извне» параметров связей на предмет нахождения системы в области устойчивости.

Помимо устойчивости, важную роль играет несмещенность стационарного выпуска относительно целевого уровня $C_{0}$, определяемого на практике, как правило, советом директоров компании. На практике информация о целевом (стратегическом) уровне выпуска, спускаясь с уровня совета директоров по управленческой вертикали вниз, искажается и размывается - и попадает на более низкие уровни управления в виде, вообще говоря, отличных от $C_{0}$ величин. В нашей простой модели акционерного общества стационарное состояние будет определяться только параметрами «акционерного контроля», но даже в этом случае нужны механизмы, позволяющие «извне» оценить величину искажений и подать совету директоров сигнал о необходимости корректировки спускаемой вниз информации о целевом выпуске.

Оценивая параметры связей в системе на предмет обеспечения устойчивости и несмещенности выпуска, компания в практических терминах оценивает систему внутреннего контроля, управления рисками и корпоративного управления. Такую оценку может проводить внешний провайдер - или обособленное подразделение (в соответствии с определением МОПП - подразделение внутреннего аудита).

Таким образом, выстраивание функционала внутреннего аудита именно в соответствии с определением МОПП позволяет построить в компании систему управления с хорошим запасом прочности, что явля- ется важнейшим фактором достижения операционных и стратегических целей организации.

Подразделению внутреннего аудита следует оценивать и, при необходимости, рекомендовать к корректировке как можно больший спектр связей в системе: соответственно, требуется его подотчетность как можно более высокому уровню управления. Отметим, что условия устойчивости, связывающие параметры системы, не накладывают требования на абсолютную величину параметров, что позволяет попадать в область устойчивости, варьируя лишь параметры связей «от совета директоров и ниже» (считая параметры связей к акционеру заранее фиксированными). Таким образом, подотчетности внутреннего аудита совету директоров в рамках модели вполне достаточно. В соответствии с МОПП внутренний аудит функционально подотчетен совету директоров компании - и теперь мы видим, что данное требование является не просто «хорошей практикой», а носит фундаментальный характер для обеспечения устойчивости компании.

\section{Список литературы}

Арнольд В.И. «Жесткие» и «мягкие» математические модели. М.: МЦНМО, 2004. - 32 с. ил.

ISBN 5-94057-134-4.

Бесекерский В.А., Попов Е.П. Теория систем автоматического регулирования. СПб.: Профессия, 2003. 752 c. ISBN: 5-93913-035-6

Кодекс корпоративного управления // Вестник Банка России: Нормативные акты и оперативная информация Центрального банка Российской Федерации. 2014. № 40 (1518). C. 3-63.

Концепция COSO Комитета спонсорских организаций комиссии Тредуэя «Внутренний контроль. Интегрированная модель» (The Commitee of Sponsoring Organization of the Treadway Commission). 2013. URL: http://www.coso.org.

Международные основы профессиональной практики внутреннего аудита. 2017. URL: http://www. iia-ru.ru/ Международные основы профессиональной практики.

Методические рекомендации по организации работы внутреннего аудита в акционерных обществах с участием Российской Федерации - 2014.

URL: http://rosim.ru/documents/226258

Методические указания по подготовке положения о внутреннем аудите - 2014. URL: http://rosim.ru/ activities/corp/methodology/documents/metod_ukaz_ norm_dok

Федеральный закон от 26.12.1995 г. № 208-Ф3 «Об акционерных обществах» (с посл. изм. и доп.). URL: http://www.consultant.ru. 
Eccles R.G., Youmans T. Materiality in Corporate Governance: The Statement of Significant Audiences and Materiality // Working paper. 2015. No. 16-023. Harvard Business School.

Ravina E., Sapienza P. What do independent directors know? Evidence from their trading. The Review of Financial Studies. 2010. No. 23 (3). P. 962-1003.

Shleifer, A., Vishny R.W. A Survey of Corporate Governance // Journal of Finance. 1997. No. 52 (2). P. 737-783.

Vernikov A. Does corporate governance really predict firms' Market values in emerging markets? The case of russian banks // Journal of corporate finance. 2013. No. 3(27). P. 21-33. 


\title{
Some fundamental factors of corporate governance system sustainability
}

\author{
Zenkov, Oleg S. \\ Certified Internal Auditor \\ The Institute of internal auditors member (https://iia-ru.ru/) \\ b. 5 str. 1, Naryshkinskaya alleya, Moscow, Russia \\ E-mail: ozenkov@mail.ru
}

\begin{abstract}
Multilevel governance systems have a number of properties that at first glance may look paradoxical. For example, additional feedback mechanism does not necessarily increase stability of the system and can even destabilize it. Developing and applying mathematical model of corporate governance allows us to identify ways to ensure company sustainability and manageability, analyze the most frequently proposed and discussed approaches to the organization of corporate governance and internal control system.

Our analysis shows drawbacks of overly strong "shareholders control": an overly active revision commission, even working professionally, increases instability of a company. One of the most important conditions for the sustainability and manageability of a company is the availability of a mechanism that ensures the delegation of decision-making to all levels of the company's management. Any strategy that implies constant improvement of performance discipline without such mechanism will sooner or later lead to a loss of the company's manageability as predicted by our dynamic model.

An essential stability for a multi-level management system could be ensured by participation of all personnel in the company's internal control system and the formation of the company's internal audit unit that could help to evaluate and improve the effectiveness of governance, risk management and control processes (the existence of an ordinary control unit providing feedback is not a fundamental factor of sustainability) as well as independence of internal audit and its functional reporting to the Board.

Typical factors that could lead to "near the border of sustainability trap" for a small state owned company are analyzed. Possible steps and their prioritization to bring the company out of this situation are discussed in this article. Approaches to the organization of the internal audit function proposed by international standards are analyzed from the standpoint of ensuring the company sustainability.
\end{abstract}

Keywords: corporate governance sustainability, multilevel governance systems, mathematical model, company manageability, internal audit, shareholders' control

JEL: G34 


\section{References}

Arnold, V.I. (2004) "Zhestkie" I "myagkie" matematicheskie modeli. [Hard and soft mathematical models]. M.: MCNMO. - 32 p. ISBN 5-94057-134-4. (in Russian)

Besekerskiy. V.A., Popov, E.P. (2003)

Teoriya system avtomaticheskogo regulirovaniya. [Theory of regulating systems] $\mathrm{SPb}$.: Professiya, 2003. - 752 p. ISBN: 5-93913-035-6. (in Russian)

COSO "Internal Control - Integrated Framework (2013)" (The Commitee of Sponsoring Organization of the Treadway Commission). URL: http://www.coso.org.

Eccles, R.G., Youmans, T. (2015) Materiality in Corporate Governance: The Statement of Significant Audiences and Materiality. Working paper, no. 16-023. Harvard Business School.

Federalnyi Zakon ot 26.12.1995 г. 208-FZ “Ob akcionernyh obshestvah" (s posl. izm. i dop.). URL: http:// www.consultant.ru. (in Russian)

International Professional Practices Framework (IPPF) (2017). URL: https://global.theiia.org/standards-guidance/ Pages/Standards-and-Guidance-IPPF.aspx
Kodeks korporativnogo upravleniya (2010). [Corporate governance code]. Vestnik Banka Rossii: Normativnye acty i operativnaya informaciya Zentralnogo banka Rossiyskoy Federacii, 40 (1518), pp. 3-63. (in Russian)

Metodicheskie recomendacii po organizacii raboty vnutrennego audita $\mathrm{v}$ akcionernyh obshestvah s uchastiem Rossiyskoy Federacii. [Recommendations on developing internal audit in government companies] - 2014. URL: http://rosim.ru/documents/226258. (in Russian)

Metodicheskie ukazaniya po podgotovke polozheniya o vnutrennem audite. [Developing internal audit chapter]. 2014. URL: http://rosim.ru/activities/corp/methodology/ documents/metod_ukaz_norm_dok. (in Russian)

Ravina, E., Sapienza, P. (2010) What do independent directors know? Evidence from their trading. The Review of Financial Studies, no. 23 (3), pp. 962-1003.

Shleifer, A., Vishny, R.W. (1997)

A Survey of Corporate Governance. Journal of Finance, no. 52 (2), pp. 737-783.

Vernikov, A. (2013) Does corporate governance really predict firms' Market values in emerging markets? The case of russian banks // Journal of corporate finance, 3(27), pp. 21-33. 